Research Article

\title{
Neural Network Supervision Control Strategy for Inverted Pendulum Tracking Control
}

\author{
Hongliang Gao ${ }^{D},{ }^{1}$ Xiaoling $\mathrm{Li}^{1},{ }^{1}$ Chao Gao, ${ }^{2}$ and Jie $\mathrm{Wu}^{1}$ \\ ${ }^{1}$ School of Electrical Engineering and Automation, Hubei Normal University, Huangshi 435002, China \\ ${ }^{2}$ The China Ship Development and Design Center, Wuhan 430064, China \\ Correspondence should be addressed to Hongliang Gao; gaohl2016@hbnu.edu.cn
}

Received 14 January 2021; Revised 17 February 2021; Accepted 10 March 2021; Published 23 March 2021

Academic Editor: Shiping Wen

Copyright $(92021$ Hongliang Gao et al. This is an open access article distributed under the Creative Commons Attribution License, which permits unrestricted use, distribution, and reproduction in any medium, provided the original work is properly cited.

This paper presents several control methods and realizes the stable tracking for the inverted pendulum system. Based on the advantages of RBF and traditional PID, a novel PID controller based on the RBF neural network supervision control method (PID-RBF) is proposed. This method realizes the adaptive adjustment of the stable tracking signal of the system. Furthermore, an improved PID controller based on RBF neural network supervision control strategy (IPID-RBF) is presented. This control strategy adopts the supervision control method of feed-forward and feedback. The response speed of the system is further improved, and the overshoot of the tracking signal is further reduced. The tracking control simulation of the inverted pendulum system under three different signals is given to illustrate the effectiveness of the proposed method.

\section{Introduction}

Inverted pendulum system has been widely investigated in the past few decades based on two important characteristics of high order and strong coupling, which are important problems in control field. And it is an unstable, nonlinear, and multivariable system. Inverted pendulum control methods have a wide range of applications in military, aerospace, robotics, and general industrial processes, such as balancing problems during robot walking, verticality issues during rocket launch, and attitude control issues during satellite flight. The RBF neural network learning control algorithm has been a hot topic in current academic research. This algorithm can solve nonlinear problems, tracking problems, and external interference problems. Therefore, using this method to study the tracking problem of the inverted pendulum is of great significance.

The RBF (radial basis function) neural network controllers of the nonlinear system are designed based on proportion integration differentiation (PID), and these methods have good control results. Here are some related research results. In [1], it is presented that RBF network to estimate complex and precise dynamics mainly solves the problem of uncertainty and external interference in the context of complex space. This method is used to solve the problem of model uncertainty and input error. In [2], a neural network adaptive control algorithm with PID is proposed. The self-learning ability and self-adaptation uncertain system dynamic characteristics are used to significantly reduce the impact of resistance disturbance on speed. The system has strong robustness under the parameter variations and external disturbances. In [3], a scheme which combines a proportion differentiation control and a RBF neural network adaptive control algorithm is used. Among them, it uses the PD control to track the trajectory of the end effector of the wire-driven parallel robot (WDPR). The RBF neural network control algorithm is applied to approximate parameters. The combination of these two methods reduces the approximation error, enhances the robustness, and improves the accuracy of the WDPR. In [4], a fuzzy logic-based offline control strategy of a single-wheeled inverted pendulum robot (SWIPR) is presented to study the error, system set time, and rise time, etc. In the end, a good control effect was achieved. In [5], RBF network is applied to reduce chatter and increase stability. In [6], it proposes a single-layer nonlinear controller to achieve that the inverted 
pendulum can be adjusted to a stable state from any initial position and achieves four degrees of freedom. Hossein [7] put forward a kind of improved PID control method based on RBF neural network. It shows that the algorithm has a better control effect than the traditional PID for the tension. In $[8,9]$, adaptive control methods are applied to the input or output of nonlinear systems. They are, respectively, applicable to a class of nonsmooth nonlinear systems and a class of multi-input multioutput nonlinear time-delay systems with input saturation. In $[10,11]$, two different adaptive methods were proposed to solve the unknown and stochastic nonlinear tracking problems of nonlinear systems. And other scholars have studied the stabilization and convergence of the system [12-14]. Kumar et al. [15] use adaptive control technology to deal with tracking problems. At present, many researchers [16-20] have conducted in-depth studies on various inverted pendulum models using different control methods.

In fact, no matter what the control system is, the system will tend to be stable under the action of the controller. Therefore, RBF neural network is used as a model for approximating uncertainty, to study error convergence, and achieved good convergence results in [21]. In [22], it is proposed to use the RBF algorithm to estimate the residual error, reduce the error through the design of the controller, increase the control signal, and obtain good heating requirements. In [23], the dynamic characteristics of machine system are explored and the characteristics of the RBF neural network are used to study the tracking problem. Under the large structure of the RBF neural network, different basis functions are selected for a comparative study to eliminate chattering in [24]. In [25], the RBF neural network implements self-feedback control, accurate prediction, and real-time control of reasonable data. It has improved tracking accuracy and estimated unmodeled dynamics and external interference issues in [26]. As more and more academic researchers understand the approximation characteristics of RBF, they add RBF neural networks to various fields to study the dynamic characteristics of different systems. It mainly solves the problems of nonlinearity, uncertainty, and external interference and uses the Lyapunov function to ensure the effectiveness of the algorithm, so that it reduces system errors and reaches a stable state [27-34].

In recent years, the footprint of PID applications can be seen in different fields. From simple PID control algorithm to complex PID algorithm control, it has played a role in different control fields. In $[35,36]$, the PID control algorithm and operation rules are studied, respectively. The characteristics of the PID algorithm are explored, and the PID has a certain degree of adaptability through simulation. According to the characteristics of the PID algorithm, some scholars have studied the tuning of the PID controller [37, 38]. Tuning PID parameters are used to optimize system performance according to actual conditions. As PID parameter tuning technology becomes more and more mature, some interested scholars use PID as a controller to study system stability and tracking issues [39-42]. In order to adapt PID to more situations, some scholars have launched the control research of fuzzy PID $[43,44]$ and fractional PID $[45,46]$. It can be seen that the PID control algorithm is a relatively classic control method.

According to the references, we can find that the RBF neural network control algorithm basically uses the Lyapunov function to determine the stability conditions. In [47], based on the nonlinear U model, RBF neural network and PD parallel control algorithm are proposed. The Lyapunov function determines the conditions of system stability, and under this condition, the tracking effect has been improved. However, from the tracking effect, the error between the system output and the tracking signal is large, and the tracking situation with external interference is not considered. Therefore, in this article, we consider these problems based on the inverted pendulum model to study its tracking problem.

In short, the control of the inverted pendulum model mainly includes three major control performances, namely, stability, accuracy, and rapidity. Then, for the tracking problem of the inverted pendulum, the three comprehensive performances also need to be considered. Therefore, we designed a supervision control method PID-RBF. We further design another supervision control strategy IPID-RBF. The stable tracking of the signal is achieved by supervision control strategy. In general, the main innovations of this paper include the following:

(1) PID-RBF strategy ensures the stability of the system. The overshoot of the system is reduced and the robustness of the system is enhanced. In the case of interference, the parameters can be adjusted adaptively to control signal tracking.

(2) IPID-RBF strategy further solves the problem of large overshoot in the control process. The adjustment time of the system is further reduced. This strategy has strong anti-interference ability, fast stability, and small error with the tracking curve.

(3) In the control process, we can use the PID-RBF strategy to replace the traditional PID control strategy. This way can make the system overshoot smaller and system stability better. IPID-RBF strategy further improves the overall performance of the system. In the IPID-RBF control strategy, the system has a faster response speed, better stability, and robustness.

The rest of the paper is organized as follows. The relevant control objective is presented in Section 2. Neural network supervision control design is presented in Section 3. The simulation study is discussed in Section 4. Finally, the conclusions are given in Section 5.

\section{Control Objective}

The inverted pendulum system can be difficult to control as the order increases. At the same time, the inverted pendulum system itself has the characteristics of complexity, instability, and nonlinearity. The inverted pendulum system is often used as an experimental project in real society. At the same time, the effectiveness of some control methods in the 


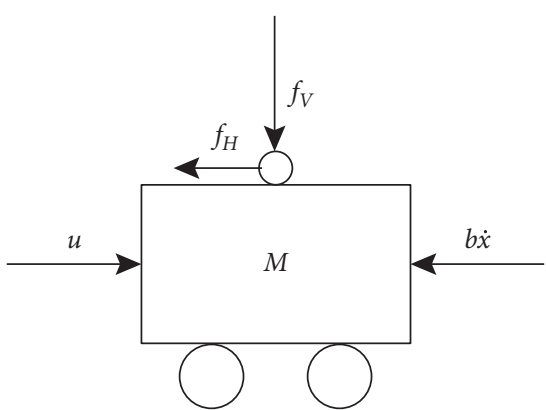

(a)

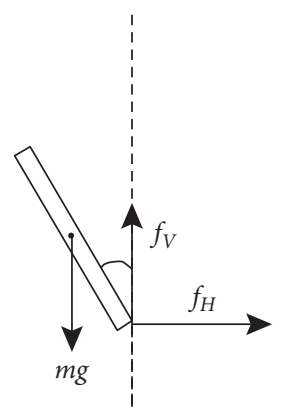

(b)
FIgURE 1: Force analysis diagram of trolley and swing rod.

introduction part has been verified by controlling the inverted pendulum system. Therefore, it has important significance for the research of inverted pendulum. In order to study the signal tracking problem, we consider the inverted pendulum model based on PID and RBF neural network control. The inverted pendulum model is similar to [48]. The force analysis of the inverted pendulum system is shown in Figure 1.

In Figure 1, the external force exerted on the trolley and its moving displacement are indicated by symbols $u$ and $x$, respectively. $\theta$ is expressed as the angle between the pendulum and the vertical direction. After differentiating its displacement is $\dot{x}$ that is the velocity of the trolley, and the friction coefficient $b$ between it and the trolley and the guide rail phase. Then we can obtain the resistance $b \dot{x}$ of the guide rail to the trolley in the horizontal direction.

In addition, the interaction between the trolley and the pendulum is decomposed into two forces perpendicular to each other in the vertical plane, where $f_{V}$ and $f_{H}$ are, respectively, used to represent the component force in the vertical and horizontal directions.

We use three motions to express the pendulum bar motion of an inverted pendulum: the horizontal motion of the center of gravity, the vertical motion of the center of gravity, and the rotation around the center of gravity. According to Newton's law of mechanics, we can get three equations of motion, $\phi=\theta+\pi$ represents the angle between the pendulum and the vertical downward direction:

$$
\begin{gathered}
f_{H}=m \frac{\mathrm{d}^{2}}{\mathrm{~d} t^{2}}(x+l \sin \phi), \\
f_{V}-m g=m \frac{\mathrm{d}^{2}}{\mathrm{~d} t^{2}}(l \cos \phi), \\
-f_{V} l \sin \phi-f_{H} l \cos \phi=I \frac{\mathrm{d}^{2} \phi}{\mathrm{d} t^{2}} .
\end{gathered}
$$

Equation (1) is equivalent to the following equation:

$$
f_{H}=m \ddot{x}+m l \ddot{\phi} \cos \phi-m l \dot{\phi}^{2} \sin \phi .
$$

Equation (2) is also equivalent to the following equation:

$$
f_{V}-m g=-m l \ddot{\phi} \sin \phi-m l \dot{\phi}^{2} \cos \phi \text {. }
$$

The resultant force in the horizontal direction of the trolley can be expressed as follows:

$$
M \ddot{x}=u-b \dot{x}-f_{H} .
$$

Put (4) into (6), the external force $u$ can be written as

$$
u=(M+m) \ddot{x}+m l \ddot{\phi} \cos \phi-m l \dot{\phi}^{2} \sin \phi+b \dot{x} .
$$

Similarly, substituting (4) and (5) into (3)yields

$$
-m l \ddot{x} \cos \phi=\left(I+m l^{2}\right) \ddot{\phi}+m g l \sin \phi .
$$

Formulas (7) and (8) are nonlinear equations of motion of vehicle-mounted inverted pendulum system. In order to facilitate the control, linearize the system. Suppose that $\theta \leq 20^{\circ}$ is within the error range of keeping stability. Because $\phi_{2}=\theta_{+2} \pi$, $\theta$ is so small; therefore, $\cos \phi \approx-1$, sin $\phi=\theta$, and $\dot{\phi}^{2}=\dot{\theta}^{2} \approx 0$. After linearization, the system is transformed into the following mathematical model:

$$
\begin{aligned}
(M+m) \ddot{x}+b \dot{x}-m l \ddot{\theta} & =u, \\
\left(I+m l^{2}\right) \ddot{\theta}-m g l \theta & =m l \ddot{x} .
\end{aligned}
$$

Taking the Laplace transform in (9), one obtains

$$
\begin{aligned}
(M+m) X(s) s^{2}+b X(s) s-m l \Phi(s) s^{2} & =U(s), \\
\left(I+m l^{2}\right) \Phi(s) s^{2}-m g l \Phi(s) & =m l X(s) s^{2} .
\end{aligned}
$$

By eliminating $X(s)$ from the equation set (10), the transfer function of the trolley to the pendulum angle is obtained as follows:

$$
G(s)=\frac{\Phi(s)}{U(s)}=\frac{(m l / q) s^{2}}{s^{4}+\left(b\left(I+m l^{2}\right) / q\right) s^{3}-((M+m) m g l / q) s^{2}-(b m g l / q) s},
$$

where $q=(M+m)\left(I+m l^{2}\right)-\left(m l^{2}\right)$ is a constant.

\section{Neural Network Supervision Control Design}

3.1. PID-RBF and IPID-RBF Control Design. Here, we introduce the PID-RBF control and IPID-RBF control. As we know, PID controller consists of three important parameters, which are proportional regulation coefficient $k_{p}$, integral regulation coefficient $k_{i}$, and differential regulation coefficient $k_{d}$. The proportional regulation coefficient $k_{p}$ can change the response speed of the system and improve the regulation precision of the system. The integral adjustment coefficient $k_{i}$ can eliminate the residual error. The dynamic performance of the system can be improved by differential adjustment coefficient $k_{d}$. As shown in Figure 2, different PID parameters have different response speeds and stability. When the response curve oscillates significantly, $k p$ should be increased, $k i$ should be increased, and $k d$ should be smaller. When the error of the response curve is large, $k p$ should be reduced, $k i$ should be reduced, and $k d$ should be increased appropriately. According to this method, the best parameters are selected to achieve the best control effect of the inverted pendulum system. 


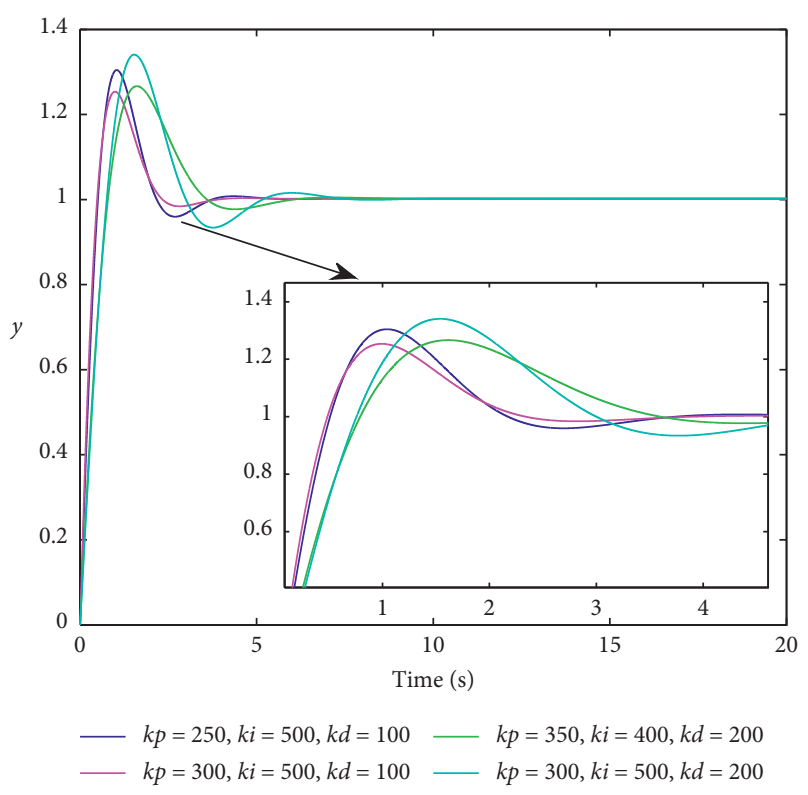

Figure 2: Step response curves under different PID parameters.

RBF neural network is a three-layer feed-forward neural network, and the mapping from input to output is linear which greatly speeds up the learning speed and avoids the problem of local minima. RBF neural network supervision control is to study the traditional controller, adjust the weight of the network online, and make the feedback control input $u_{p}(k)$ tend to zero. The structure of the RBF neural network supervision control system is shown in Figure 3.

In the RBF network structure, the input signal of the network is taken as $r(k), H=\left[h_{1}, \ldots, h_{m}\right]^{T}$ is radial basis function vector, and Gaussian basis function is $h_{j}$ which is expressed as follows:

$$
h_{j}=\exp \left(-\frac{\left\|r(k)-C_{j}\right\|^{2}}{2 b_{j}^{2}}\right),
$$

where $j=1, \ldots, m, b_{j}$ is the base width parameter of node $j$, $b_{j}>0, C_{j}$ is the center vector of node $j, C_{j}=\left[c_{11}, \ldots, c_{1 m}\right]^{T}$, and $b_{j}=\left[b_{1}, \ldots, b_{m}\right]^{T}$.

The weight vector of the network is given by

$$
W=\left[w_{1}, \ldots, w_{m}\right]^{T} .
$$

The output of RBF network is denoted by

$$
u_{n}(k)=h_{1} w_{1}+\cdots h_{j} w_{j}+\cdots+h_{m} w_{m}
$$

where $m$ is the number of hidden layer neurons in the network.

The control law is given by

$$
u(k)=u_{p}(k)+u_{n}(k)
$$

The performance indicators of the neural network adjustment are given by

$$
E(k)=\frac{1}{2}\left(u_{n}(k)-u(k)\right)^{2} .
$$

The approximation is as follows:

$$
\frac{\partial u_{p}(k)}{\partial w_{j}(k)} \approx \frac{\partial u_{n}(k)}{\partial w_{i}(k)} \text {. }
$$

The error caused by the approximation is compensated by weight adjustment. The gradient descent method is adopted to adjust the weights of the network.

$$
\begin{aligned}
\Delta w_{j}(k) & =-\eta\left(u_{n}(k)-u(k)\right) h_{j}(k) \\
& =-\eta \frac{\partial E(k)}{\partial w_{j}(k)} h_{j}(k)
\end{aligned}
$$

where $\eta$ is the learning rate.

$\alpha$ is the momentum factor, and we get the adjustment process of neural network weights as follows:

$W(k)=W(k-1)+\Delta W(k)+\alpha(W(k-1)-W(k-2))$.

(1) PID controller based on the RBF neural network supervision control method (PID-RBF) includes error signal $e_{1}(k)$, cumulative error signal $\sum_{s=0}^{k} e_{1}(s)$, current error, and last time error difference signal $\Delta e_{1}(k)=\left(e_{1}(k)-e_{1}(k-1)\right)$ processing.

The PID-RBF control law can be expressed as follows:

$$
\bar{u}_{p}(k)=k_{p} * e_{1}(k)+k_{i} * T_{1} * \sum_{s=0}^{k} e_{1}(s)+\frac{k_{d}}{T_{1}} *\left(e_{1}(k)-e_{1}(k-1)\right) .
$$

The network input vector of the PID-RBF control is given by

$$
\begin{aligned}
X(k) & =\left[x_{1}(k), x_{2}(k), x_{3}(k)\right]^{T} \\
& =\left[\begin{array}{c}
e_{1}(k) \\
\sum_{s=0}^{k} e_{1}(s)\left(e_{1}(k)-e_{1}(k-1)\right)
\end{array}\right],
\end{aligned}
$$

where $T_{1}$ represents sampling time, $e_{1}(k)=r(k)-y_{1}(k)$, the input signal is $r(k)$, and $y_{1}(k)$ is the output sequence of the system response, $s=0,1, \ldots, k$.

The network output of the PID-RBF control is

$$
\bar{u}_{n}(k)=h_{1} \bar{w}_{1}+\cdots h_{j} \bar{w}_{j}+\cdots+h_{m} \bar{w}_{m}
$$

According to (15), (20), and (22), we can express its total control law as 


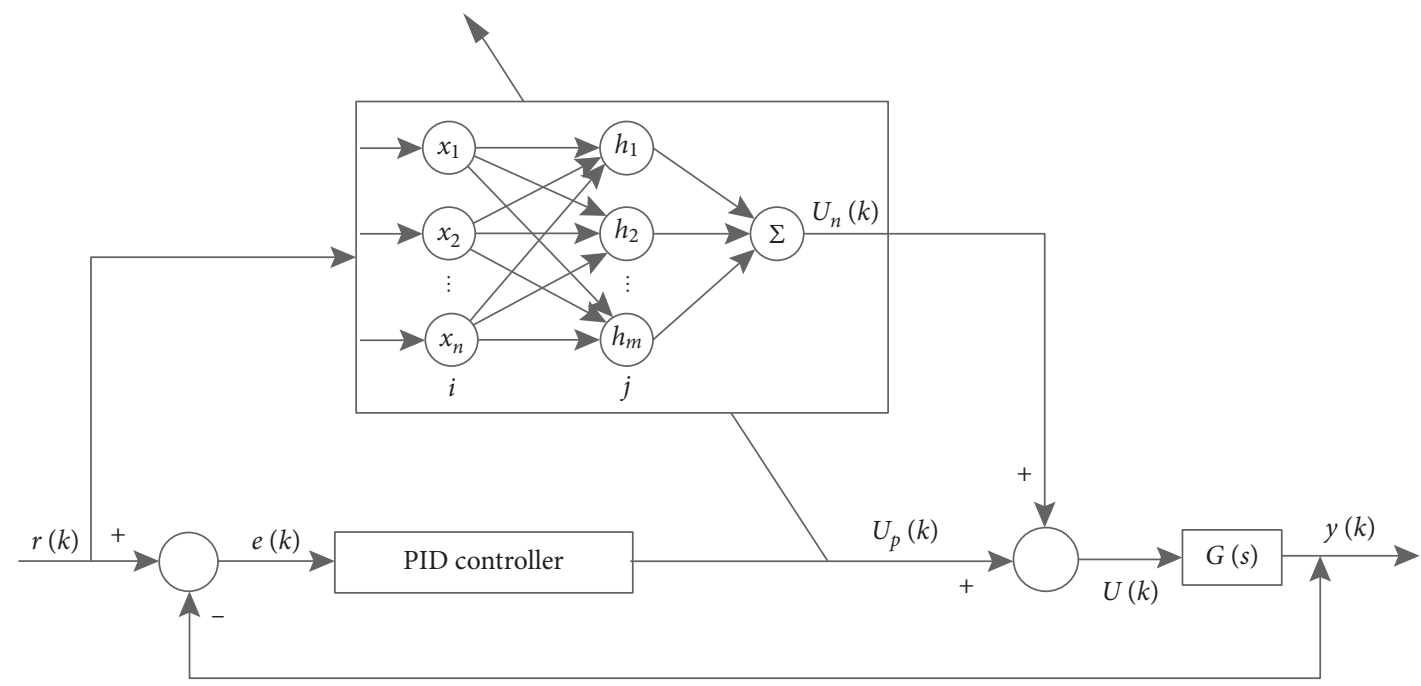

FIGURE 3: Structure of neural network supervision control system.

$$
\begin{gathered}
\bar{u}(k)=\bar{u}_{p}(k)+\bar{u}_{n}(k) \\
=k_{p} * e_{1}(k)+k_{i} * T_{1} * \sum_{s=0}^{k} e_{1}(s)+\frac{k_{d}}{T_{1}} *\left(e_{1}(k)-e_{1}(k-1)\right)+h_{1} \bar{w}_{1}+\cdots h_{j} \bar{w}_{j}+\cdots+h_{m} \bar{w}_{m} .
\end{gathered}
$$

The weight adjustment process of RBF neural network supervision is expressed as

$$
\begin{aligned}
\Delta \bar{w}_{j}(k)= & -\eta\left(\bar{u}_{n}(k)-\bar{u}(k)\right) h_{j}(k)=-\eta \frac{\partial \bar{E}(k)}{\partial \bar{w}_{j}(k)} h_{j}(k), \\
\bar{w}_{j}(k)= & \bar{w}_{j}(k-1)+\Delta \bar{w}_{j}(k)+\alpha\left(\bar{w}_{j}(k-1)-\bar{w}_{j}(k-2)\right) \\
= & \bar{w}_{j}(k-1)-\eta\left(\bar{u}_{n}(k)-\bar{u}(k)\right) h_{j}(k) \\
& +\alpha\left(\bar{w}_{j}(k-1)-\bar{w}_{j}(k-2)\right) .
\end{aligned}
$$

(2) An improved PID controller based on RBF neural network supervision control strategy (IPID-RBF) includes error signal $e_{2}(k)$, cumulative error signal $\tilde{e}_{2}(k)$, current error, and last time error difference signal $\Delta e_{2}(k)=e_{2}(k)-e_{2}(k-1)$ processing.

$\tilde{u}_{p}(k)=k_{p} * e_{2}(k)+k_{i} * T_{2} * \widetilde{e}_{2}(k)+\frac{k_{d}}{T_{2}} *\left(e_{2}(k)-e_{2}(k-1)\right)$.
The network input vector of the IPID-RBF control is

$$
\tilde{X}_{1}(k)=\left[\tilde{x}_{1}(k), \tilde{x}_{2}(k), \tilde{x}_{3}(k)\right]^{T}=\left[\begin{array}{c}
e_{2}(k) \\
\tilde{e}_{2}(k) \\
\left(e_{2}(k)-e_{2}(k-1)\right)
\end{array}\right] \text {, }
$$

where $y_{2}(k)$ is the output sequence of the system response, $e_{2}(k)=r(k)-y_{2}(k), \tilde{e}_{2}(k)=\left(\left(e_{2}(0) * T_{2}+e_{2}(1)\right) * T_{2}+\right.$ $\left.\cdots+e_{2}(k-1)\right) * T_{2}+e_{2}(k)$, and $T_{2}$ represents sampling time.

The network output of the IPID-RBF control is as follows:

$$
\widetilde{u}_{n}(k)=h_{1} \widetilde{w}_{1}+\cdots h_{j} \widetilde{w}_{j}+\cdots+h_{m} \widetilde{w}_{m} .
$$

According to (15), (25), and (27), we can express its total control law as

$$
\begin{aligned}
\tilde{u}(k) & =\widetilde{u}_{p}(k)+\widetilde{u}_{n}(k) \\
& =k_{p} * e_{2}(k)+k_{i} * T_{2} * \widetilde{e}(k)+\frac{k_{d}}{T_{2}} *\left(e_{2}(k)-e_{2}(k-1)\right)+h_{1} \widetilde{w}_{1}+\cdots h_{j} \widetilde{w}_{j}+\cdots+h_{m} \widetilde{w}_{m} .
\end{aligned}
$$


The weight adjustment process of RBF neural network supervision is expressed as

$$
\begin{aligned}
\Delta \widetilde{w}_{j}(k)= & -\eta\left(\widetilde{u}_{n}(k)-\tilde{u}(k)\right) h_{j}(k)=-\eta \frac{\partial \widetilde{E}(k)}{\partial \widetilde{w}_{j}(k)} h_{j}(k), \\
\widetilde{w}_{j}(k)= & \widetilde{w}_{j}(k-1)+\Delta \widetilde{w}_{j}(k)+\alpha\left(\widetilde{w}_{j}(k-1)-\widetilde{w}_{j}(k-2)\right) \\
= & \widetilde{w}_{j}(k-1)-\eta\left(\widetilde{u}_{n}(k)-\tilde{u}(k)\right) h_{j}(k) \\
& +\alpha\left(\widetilde{w}_{j}(k-1)-\widetilde{w}_{j}(k-2)\right) .
\end{aligned}
$$

3.2. Control Algorithm Stability Analysis. For the stability analysis of the control algorithm, we fully consider the performance indicators adjusted by the neural network $E(k)$, equation (16) $E(k)=(1 / 2)\left(u_{n}(k)-u(k)\right)^{2}$. The equation of state for the free motion of a linear stationary discrete system is

$$
x(k+1)=G x(k)
$$

The discrete Lyapunov equation is

$$
G^{T} P G-P=-Q,
$$

where $G$ is system matrix and $P$ and $Q$ are positive definite real symmetric matrices.

The Lyapunov function is expressed as

$$
V(x)=x^{T} P x .
$$

When $V(0)=0, x$ is the solution of the following state equation [47]:

$$
x(k+1)=x^{\prime}(k) P x(k)=G x(k) .
$$

The increment of the Lyapunov function is

$$
\Delta V[x(k)]=V[x(k+1)]-V[x(k)] .
$$

The PID-RBF control function $V_{1}[x(k)]=(1 / 2) \sum_{i=0}^{k} \bar{e}_{1}^{2}(i)$. From equation (34), $\Delta V_{1}[x(k)]$ can be expressed as

$$
\begin{aligned}
\Delta V_{1}[x(k)] & =V_{1}[x(k+1)]-V_{1}[x(k)] \\
& =\frac{1}{2}\left(\sum_{i=0}^{k+1} \bar{e}_{1}^{2}(i)-\sum_{i=0}^{k} \bar{e}_{1}^{2}(i)\right)=\frac{1}{2} \sum_{i=0}^{k}\left(\bar{e}_{1}^{2}(i+1)-\bar{e}_{1}^{2}(i)\right)=\frac{1}{2} \sum_{i=0}^{k}\left(\left[\bar{e}_{1}(i)+\Delta \bar{e}_{1}(i)\right]^{2}-\bar{e}_{1}^{2}(i)\right)=\frac{1}{2} \sum_{i=0}^{k}\left(2 \bar{e}_{1}(i) \cdot \Delta \bar{e}_{1}(i)+\bar{e}_{1}^{2}(i)\right) .
\end{aligned}
$$

Combining the previous error and weight adjustment methods, we can express $e_{1}(k+1)$ as

$$
\bar{e}_{1}(k+1)=\bar{e}_{1}(k)+\Delta \bar{e}_{1}(k)=\bar{e}_{1}(k)+\left(\frac{\partial \bar{e}_{1}(k)}{\partial \bar{w}(k)}\right) \cdot \Delta \bar{w}(k) .
$$

The gradient descent method is used to adjust the weight of the network which can be rewritten as

$$
\Delta \bar{w}_{j}(k)=-\eta \frac{\partial \bar{E}(k)}{\partial \bar{w}_{j}(k)} h_{j}(k)=-\eta \frac{\partial \bar{E}(k)}{\partial \bar{e}_{1}(k)} \frac{\partial \bar{e}_{1}(k)}{\partial \bar{w}_{j}(k)} h_{j}(k) .
$$

Combining equations (36) and (37) and performance indicators $\bar{E}(k)=(1 / 2)\left(\bar{u}_{n}(k)-\bar{u}(k)\right)^{2}=(1 / 2) \bar{e}_{1}^{2}(k)$, we can get the following equation:

$$
\begin{aligned}
\Delta \bar{e}_{1}(k) & =\left(\frac{\partial \bar{e}_{1}(k)}{\partial \bar{w}(k)}\right)^{T} \times \Delta \bar{w}(t) \\
& =\left(\frac{\partial \bar{e}_{1}(k)}{\partial \bar{w}(k)}\right)^{T} \times\left(-\eta \frac{\partial \bar{E}(k)}{\partial \bar{e}_{1}(k)} \frac{\partial \bar{e}_{1}(k)}{\partial \bar{w}(k)} h_{j}(k)\right) \\
& =-\eta h_{j}(k) G_{1} G_{1}^{T} \cdot \bar{e}_{1}(k),
\end{aligned}
$$

where $G_{1}=\left(\partial \bar{e}_{1}(k) / \partial \bar{w}(k)\right)$.
According to equation (38), (35) can be expressed as follows:

$$
\begin{aligned}
\Delta V_{1}[x(k)]= & -\frac{1}{2} \sum_{i=0}^{k}\left(G_{1}^{T} \cdot \bar{e}_{1}(k)\right)^{T} \\
& \times\left(2 \eta h_{j}-\eta^{2} h_{j}^{2} G_{1} G_{1}^{T}\right)\left(G_{1}^{T} \cdot \bar{e}_{1}(k)\right) .
\end{aligned}
$$

When the value field of $\eta h_{j}$ is $0<\eta h_{j}<2\left(G_{1} G_{1}^{T}\right)^{-1}$, we can get $2 \eta h_{j}-\eta^{2} h_{j}^{2} G_{1} G_{1}^{T}>0$. We analyse equation (39) and find that the product of $\left(G_{1}^{T} \cdot \bar{e}_{1}(k)\right)^{T}$ and $G_{1}^{T} \cdot \bar{e}_{1}(k)$ is positive. Therefore, we can conclude $\Delta V_{1}[x(k)]<0$. When $\Delta V_{1}[x(k)]<0$, the system is stable. Because of $V_{1}[x(k)]=(1 / 2) \sum_{i=0}^{k} \bar{e}_{1}^{2}(i)$, the relation between the preceding and the following is expressed as $(1 / 2) \bar{e}_{1}^{2}(k+1)<(1 / 2) \bar{e}_{1}^{2}(k)$, and then $\left|\bar{e}_{1}(k+1)\right|<\left|\bar{e}_{1}(k)\right|$, $\lim _{k \rightarrow \infty} \bar{e}_{1}(k)=0$. Therefore, the control algorithm is convergent.

\section{Simulation Studies}

This section provides some simulations to show the inverted pendulum tracking effect of PID-RBF supervision control and IPID-RBF supervision control. We consider the inverted pendulum model and take the swing angle of the swing rod as the controlled object. Under zero initial conditions, 
$M=1.096, m=0.109, \quad l=0.25, g=9.8, I=0.0034$, and $b=0.1$. The transfer function can be expressed as

$$
G(s)=\frac{2.3566 s^{2}}{s^{4}+0.0883 s^{3}-27.8285 s^{3}-2.3094 s} .
$$

The transfer function of the inverted pendulum system is discretized by $z$ transformation. The discretized object after $z$ transformation is

$$
\begin{array}{r}
y(k)=-\operatorname{den}(2) * y(k-1)-\operatorname{den}(3) * y(k-2)-\operatorname{den}(4) * y(k-3) \\
+\operatorname{num}(2) * u(k-1)+\operatorname{num}(3) * u(k-2)+\operatorname{num}(4) * u(k-3) .
\end{array}
$$

The base width parameter is given by

$$
c=[-2,-1,1,2]^{T} \text {. }
$$

The center vector is as follows:

$$
b=[0.5,0.5,0.5,0.5]^{T} \text {. }
$$

We consider $k_{p}=300, k_{d}=100, k_{i}=500, t s=0.001$, $\eta=0.30$, and $\alpha=0.05$.

In Figure 4, the chart is given a square wave signal using neural network supervision control and traditional PID control. Its amplitude is one. From the diagram, it can be seen that the amplitude oscillation of the neural network supervision control is smaller than that of the traditional PID controller. The IPID-RBF supervision control tends to be stable, fastest, and more gentle. Obviously, PID-RBF and IPID-RBF supervision control have stable speed and high accuracy compared with pure PID control.

In Figure 5, the graph shows the RBF neural network supervision control tracking the input square wave signal parameter curve, the $u_{n}$ is the RBF network supervision control online learning adjustment curve, and the $u_{p}$ is the PID adjustment curve in the RBF network supervision control; superposed curves $u$ are the sum of $u_{n}$ and $u_{p}$ in RBF networks supervised control. Comparing two adjustments of three curves, because of the transformation of square wave signal from positive to negative, the value of RBF network supervision control online learning adjusting curve is changed from zero to positive, the PID adjusting curve in $\mathrm{RBF}$ network supervision control is negative at the jump instant, and the variation of the superposition curve is relatively smooth.

In Figure 6, the diagram is given step signal using RBF neural network supervision control and traditional PID control. Its amplitude is one. From the diagram, the amplitude oscillation of the RBF network supervisory control is smaller than that of the conventional PID control. PID-RBF supervision control is stable after $3.8 \mathrm{~s}$. IPID-RBF supervision control is stable after $2.5 \mathrm{~s}$. Obviously, IPID-RBF supervision control is more stable and accurate.

In Figure 7, the graph shows the RBF neural network supervision control tracking the input parameter curve of the step signal. RBF neural network supervision control online learning adjustment curve $u_{n}$ and superposition curve $u$ change trend are basically consistent.

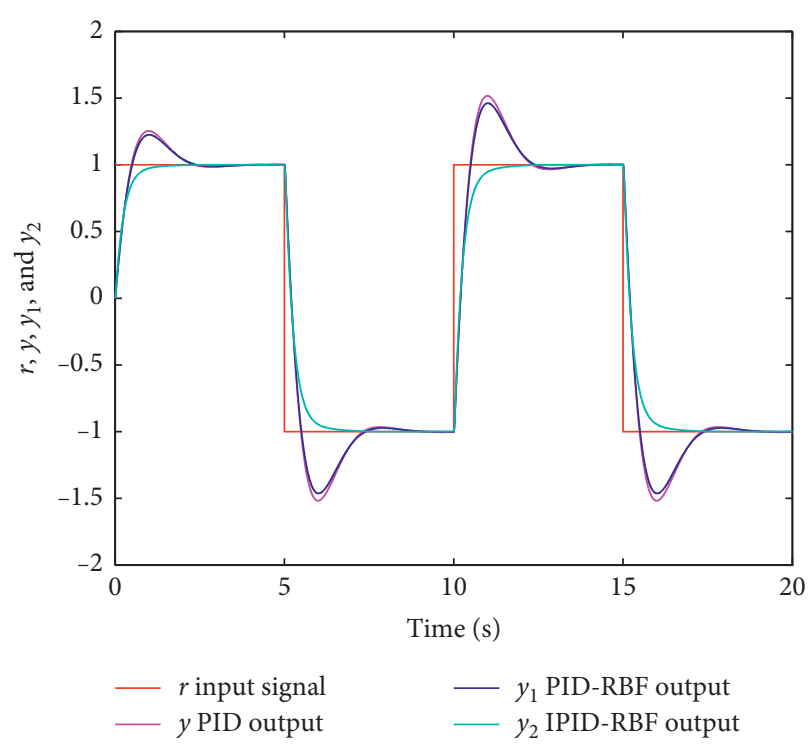

FIgURE 4: Square wave tracking.

In Figure 8, the graph is given sine wave signal $r$ using RBF neural network supervision control and traditional PID control to track. Its amplitude is one. As we can see from the picture, the amplitude oscillation of the RBF network supervision control is smaller than that of the pure PID, and the effect of the RBF neural network supervision control is better than that of pure PID control in the time period from 0 s to $20 \mathrm{~s}$. There is no error coincidence between the RBF neural network supervision control curve $\left(y_{1}\right.$ or $\left.y_{2}\right)$ and the $r$ curve. The RBF network supervision control has less error and better accuracy than the pure PID control. It can be clearly observed from the partially enlarged view that the IPID-RBF control has the highest coincidence and the best tracking effect.

In Figure 9, the diagram shows the RBF neural network supervision control tracking the parameter curve of the input sine wave signal. In RBF network supervision control, the change of PID regulating curve $u_{p}$ is small, and the change of $u$ in RBF network supervision control is gentle.

From Figures 8 and 9, the RBF neural network supervision control online learning adjustment curve $u_{n}$ and the input signal $r$ show the opposite trend change under the input sine wave signal. As the input signal increases, the adjustment curve $u_{n}$ decreases. The input signal $r$ decreases, and the adjustment curve $u_{n}$ increases. The adjustment trend of $u_{n}$ is related to the change of weight $w$. In the process of adjustment, the value of $h$ is positive, $u_{n}=\sum_{j=1}^{j=m} h_{j} w_{j}$. When the value of the input signal is positive, the weight $w$ changes $\sum_{j=1}^{j=m} h_{j} w_{j}$ correspondingly and the value is negative; when the value of the input signal is negative, the weight $w$ changes $\sum_{j=1}^{j=m} h_{j} w_{j}$ correspondingly and the value is positive.

Figure 10 shows that, for a given step signal with an amplitude of one, a given pulse-type disturbance amplitude is one. Interference time is $0.5 \mathrm{~s}$. The pure PID and the RBF neural network supervision control are used for tracking, respectively. The disturbance is added when the time is $5 \mathrm{~s}$ in Figure 10. And the amplitude oscillation of the RBF network 


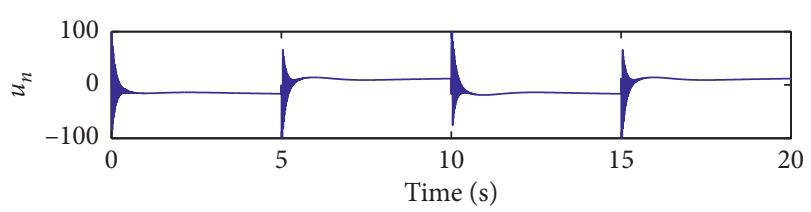

(a)

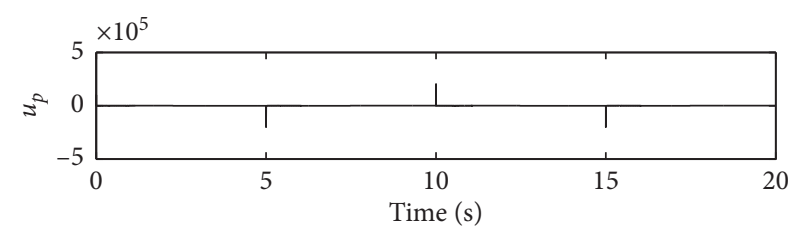

(b)

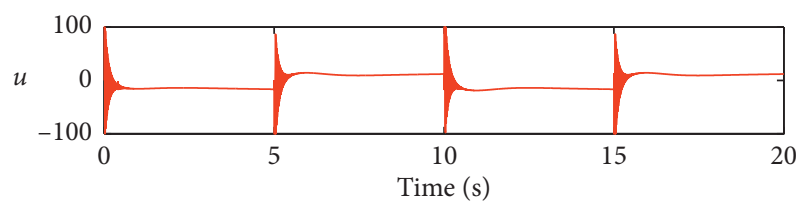

(c)

FIGURE 5: Square wave tracking RBF control parameter.

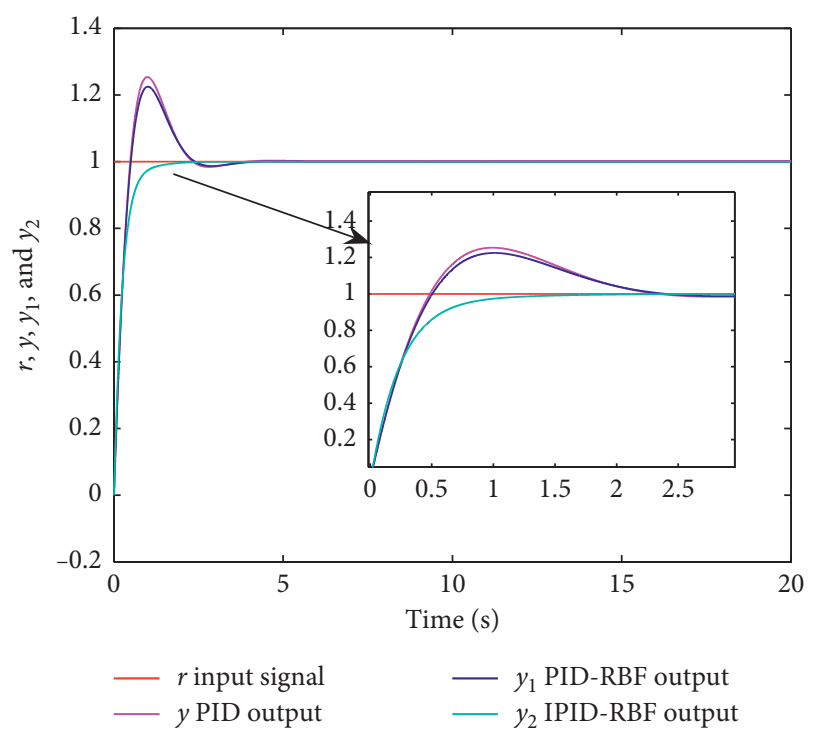

FIGURE 6: Step signal tracking.

supervision control is smaller than that of the pure PID control when the disturbance appears. Then, the system can adjust to the stable state quickly after the disturbance disappears to realize the step signal tracking.

Figure 11 shows the RBF neural network supervision control with known disturbance signal and step signal input. There are three large-scale adjustments in total: the adjustment of the system stability before the disturbance is added, the adjustment of the error caused by the disturbance compensation, and the readjustment of the system stability. In RBF network supervision control, the curves $u_{n}$ and $u$ tend to be stable after online learning.

In Figure 12, the graph is given a square wave signal. Its signal amplitude is one. Given pulse-type disturbance

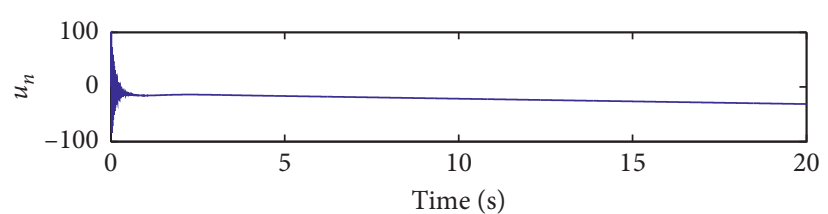

(a)

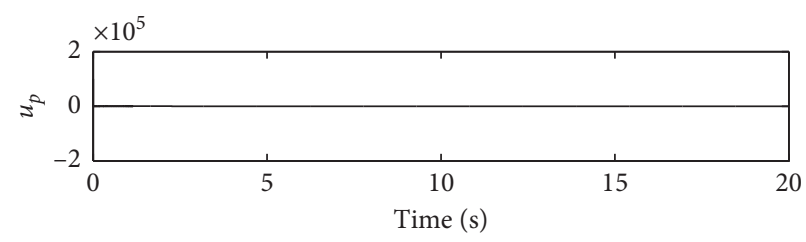

(b)

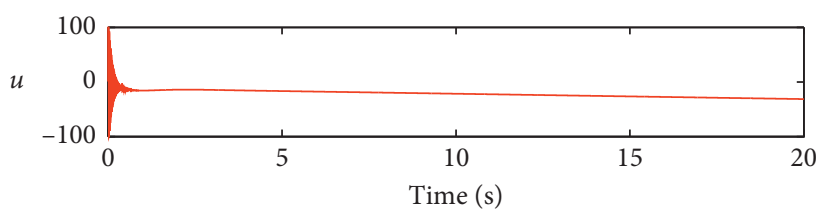

(c)

FigURE 7: Square wave tracking RBF control parameter.

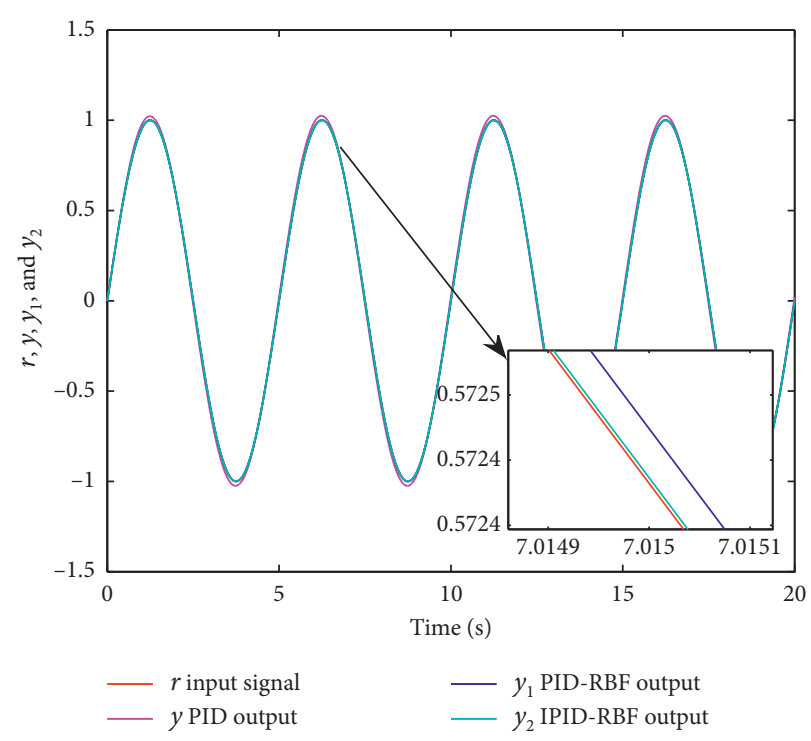

FIgURE 8: Sine wave tracking.

amplitude is two, and the time duration is $0.5 \mathrm{~s}$. When time is $5 \mathrm{~s}$, the disturbance is added. When the disturbance appears, the amplitude oscillation of RBF network supervision control is smaller than that of the pure PID. It is observed in the picture that the system quickly adjusts to the stable state without oscillation and realizes the square wave signal tracking under the IPID-RBF control.

In Figure 13, the picture shows the RBF neural network supervision control with the input of square wave signal and known disturbance signal. In the diagram, there are three large-scale adjustments between 0 and 10 seconds, such as the adjustment of system stability before the disturbance is added, the adjustment of the error caused by disturbance compensation, and the adjustment of system stability. 


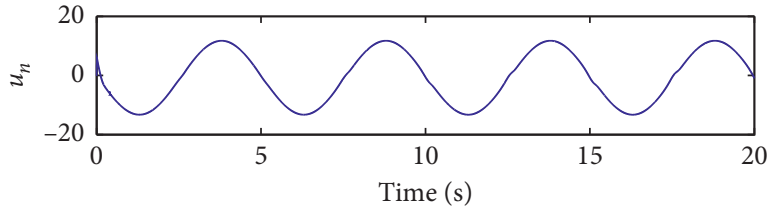

(a)

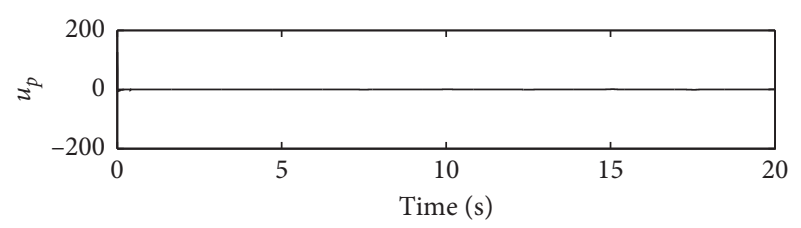

(b)

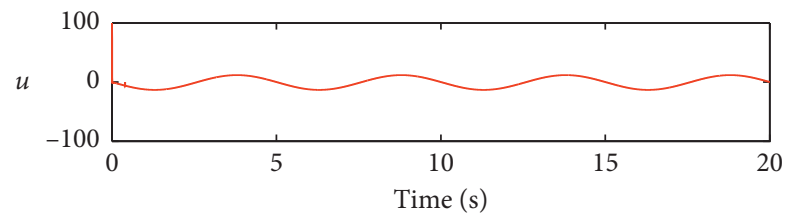

(c)

FIGURE 9: Sine wave tracking RBF control parameter

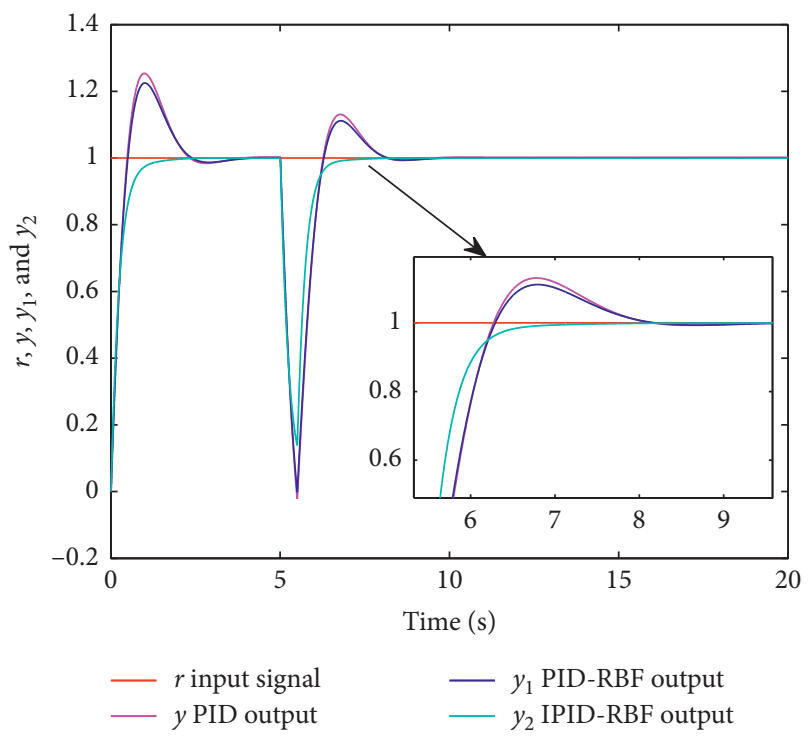

Figure 10: Step signal tracking.

Combined with the regulation of compensating disturbance in the case of step input, traditional PID and the RBF neural network supervision control are used in the regulation of compensating disturbance for the time of $0.5 \mathrm{~s}$. After $0.5 \mathrm{~s}$, the stability of the system is adjusted to stable tracking signal. Among them, the IPID-RBF control had the least amount of speed to adjust.

In Figure 14, the graph is given sine wave signal. Its signal amplitude is one. Given pulse-type disturbance amplitude is two, and the time duration is $0.5 \mathrm{~s}$. When time is $5 \mathrm{~s}$, the disturbance is added. It can be seen that the deviation of $y_{1}$ or $y_{2}$ from $r$ is less than that of the pure PID, and the curve of RBF neural network supervision control has a higher coincidence with the given sine signal after the disturbance disappears.

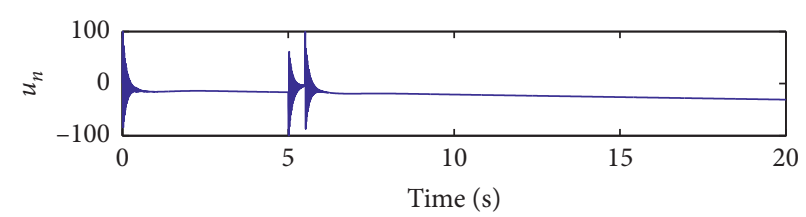

(a)

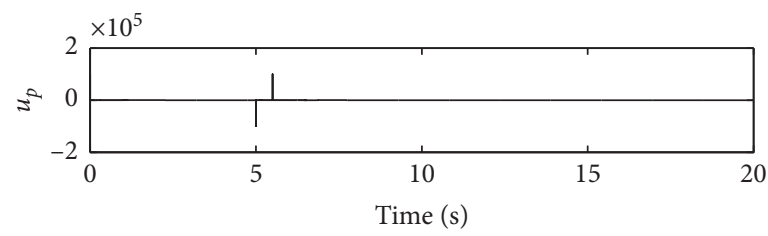

(b)

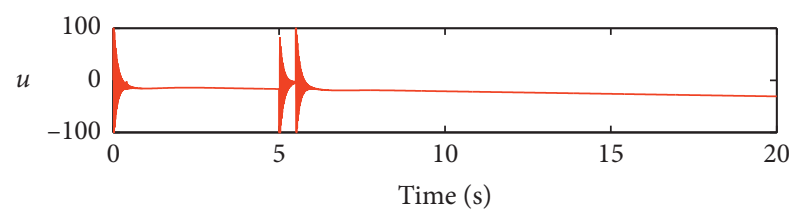

(c)

FIGURE 11: Step signal tracking RBF control parameter.

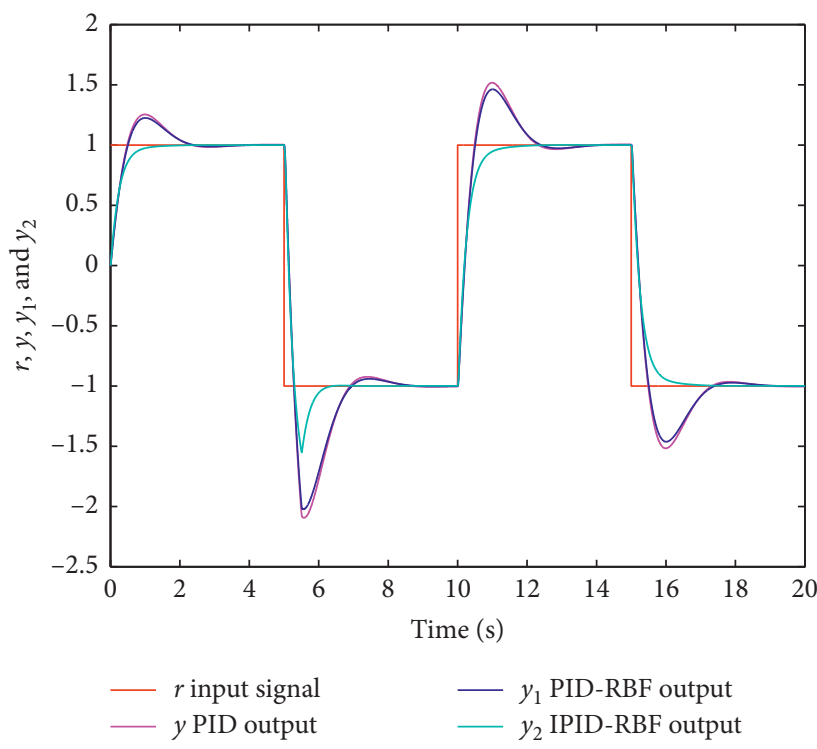

Figure 12: Square wave tracking.

Figure 15 shows that the disturbance amplitude is given to one, and the time duration is $0.5 \mathrm{~s}$, which is monitored by $\mathrm{RBF}$ network with the input of sine wave. Compared with the unperturbed condition, the value of the online learning adjustment curve $u_{n}$ of RBF neural network supervision control is larger. There are three large pulse-type jumps of $u_{n}$ and $u_{p}$ superposed curves $u$ in RBF network supervisory control. The other time RBF network supervision control online learning adjustment curve $u_{n}$ and the RBF neural network supervision control superposition curve $u$ change trend are basically consistent.

Combining Table 1 and the error graphs from Figures 16 to 21 , we can find that the traditional PID control has the worst effect, long adjustment time, and greater error than the 


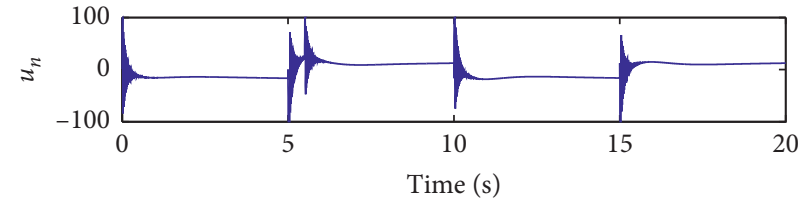

(a)

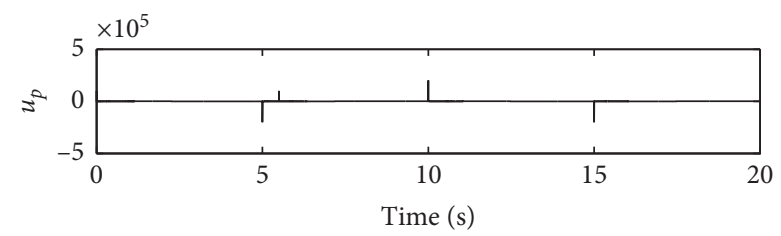

(b)

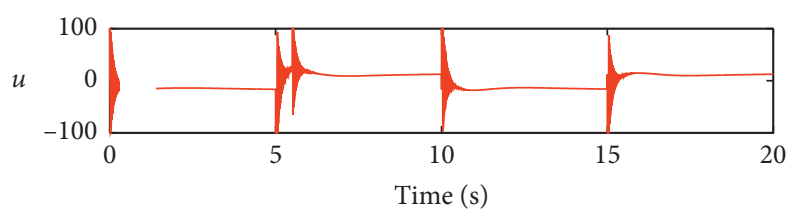

(c)

FIGURE 13: Square wave tracking RBF control parameter.

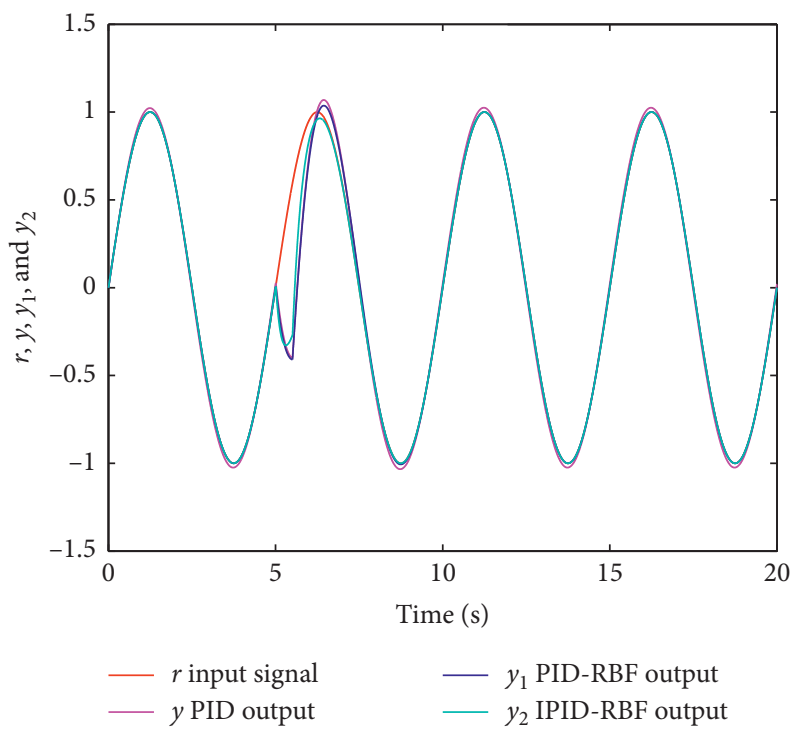

FIGURE 14: Sine wave tracking.

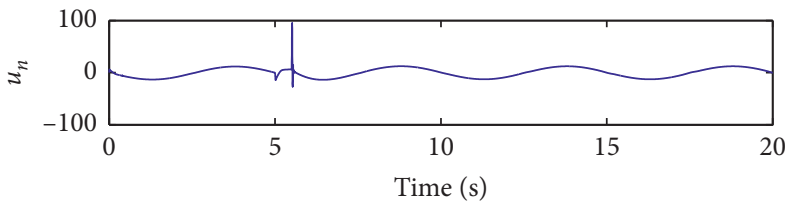

(a)

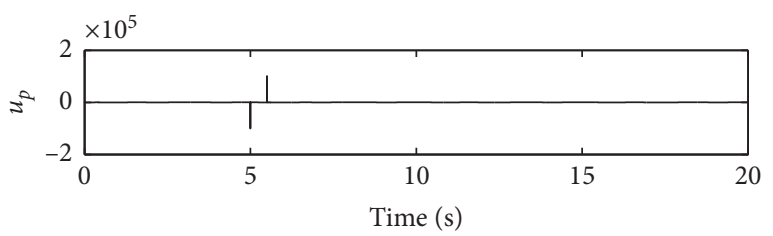

(b)

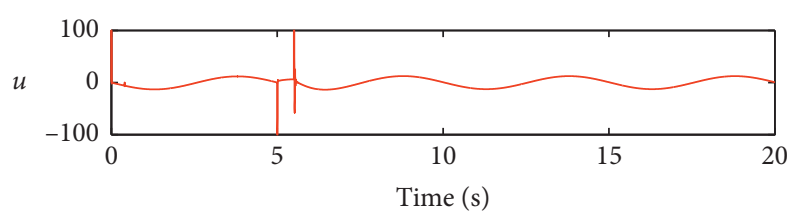

(c)

FIgURE 15: Sine wave tracking RBF control parameter. 
TABLE 1: The values of error when $k=20000$.

\begin{tabular}{|c|c|c|c|c|c|}
\hline Serial number & Disturbance & Input signal & $e(k)$ & $e_{1}(k)$ & $e_{2}(k)$ \\
\hline (1) & Inexistence & Step signal & -0.0020 & $-1.6054 e-13$ & 0.0013 \\
\hline (2) & Existence & Step signal & -0.0020 & $2.4204 e-10$ & 0.0013 \\
\hline (3) & Inexistence & Sine signal & -0.0202 & $8.2377 e-4$ & $8.6502 e-4$ \\
\hline (4) & Existence & Sine signal & -0.0202 & $7.9602 e-4$ & $8.4492 e-4$ \\
\hline (5) & Inexistence & Square signal & 0.0040 & 0.0014 & -0.0013 \\
\hline (6) & Existence & Square signal & 0.0040 & 0.0014 & -0.0014 \\
\hline
\end{tabular}

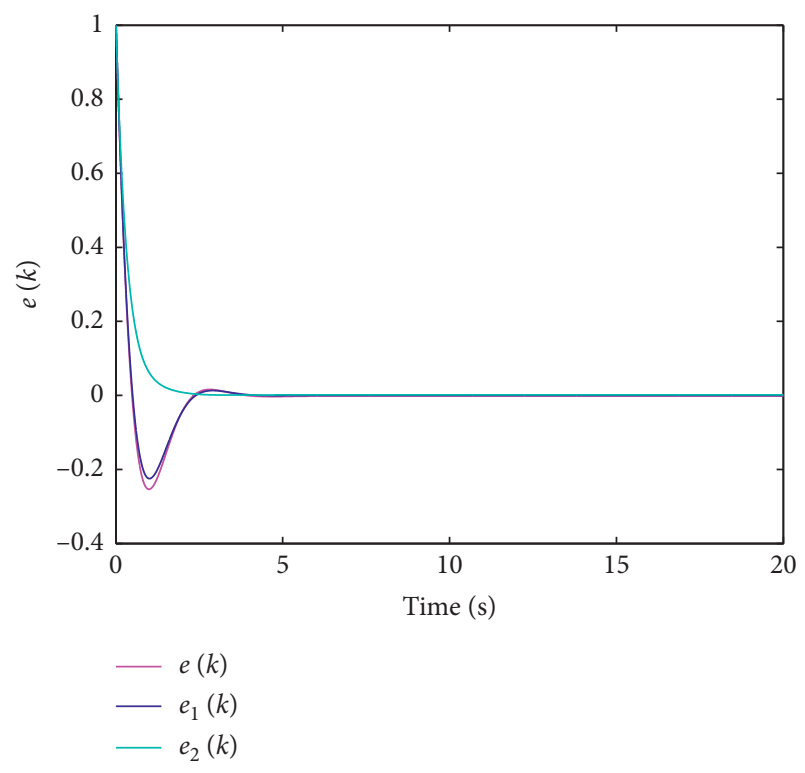

FIGURE 16: Step signal tracking error without interference.

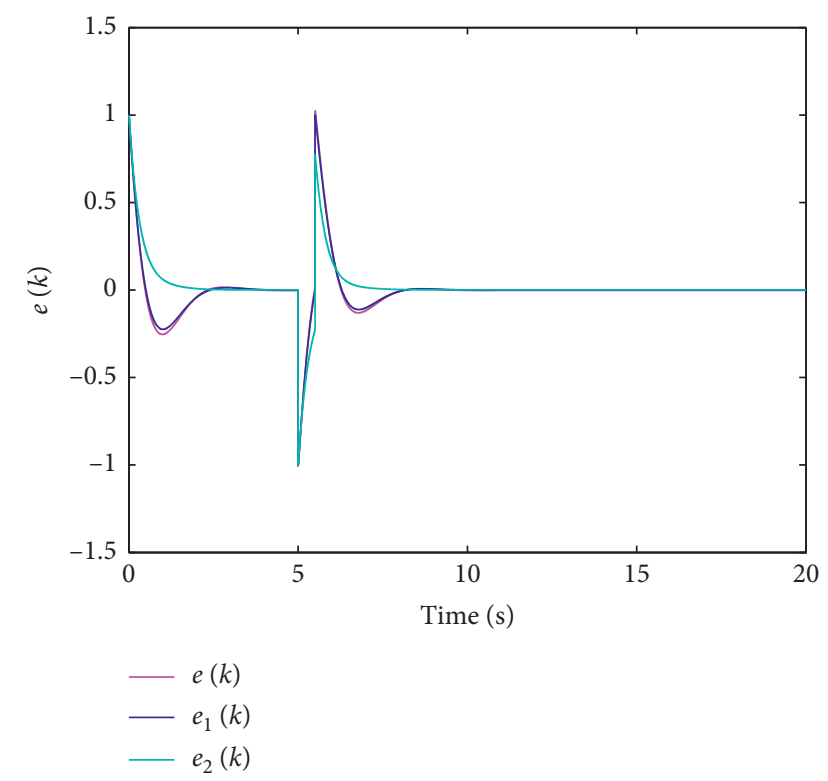

FIGURE 17: Step signal tracking error under interference.

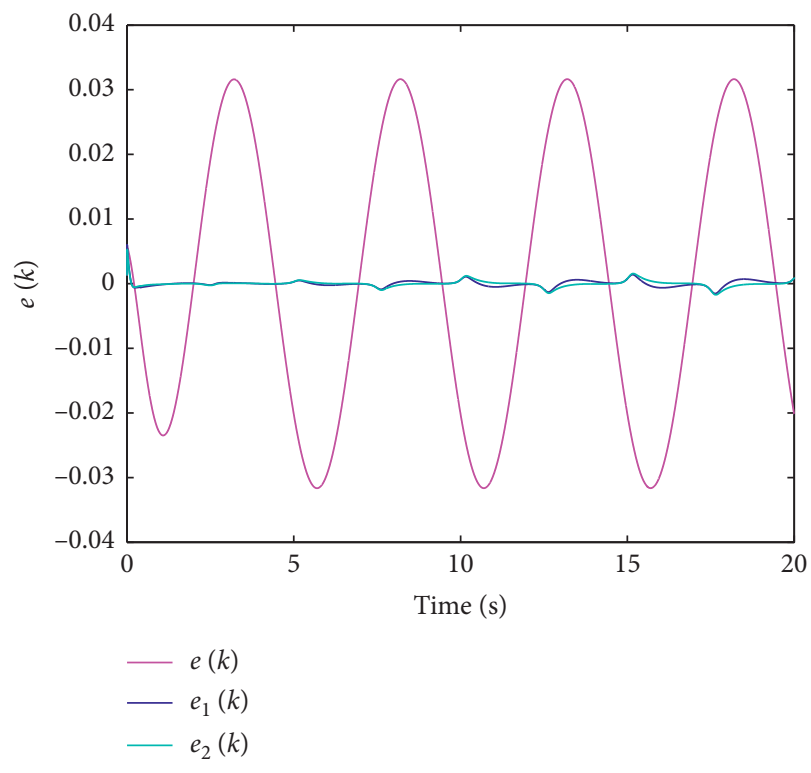

FIGURE 18: Sinusoidal signal tracking error without interference.

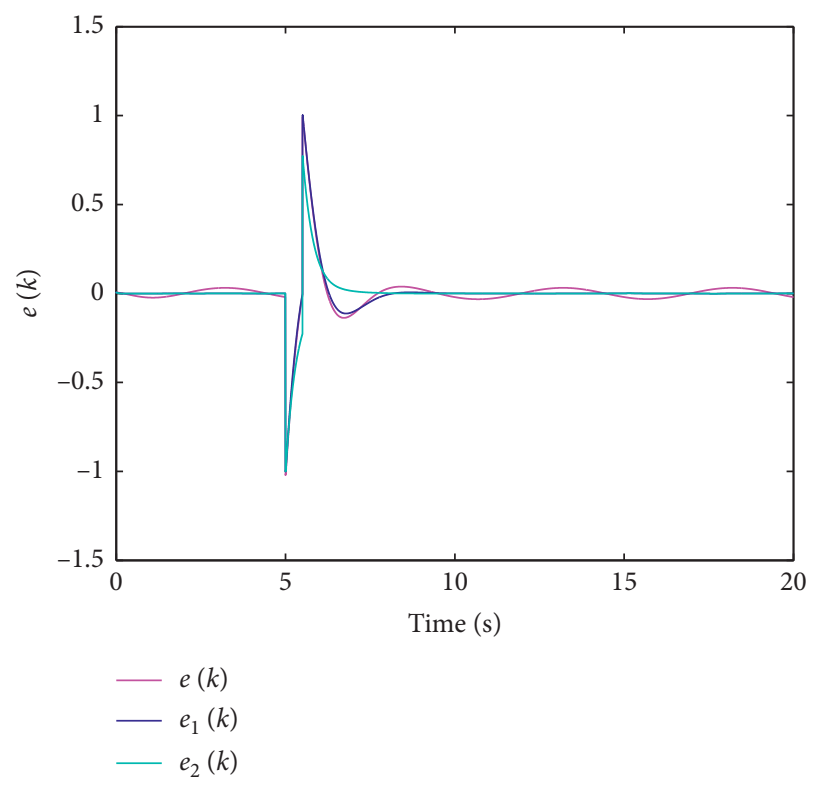

FIGURE 19: Sinusoidal signal tracking error under interference. 


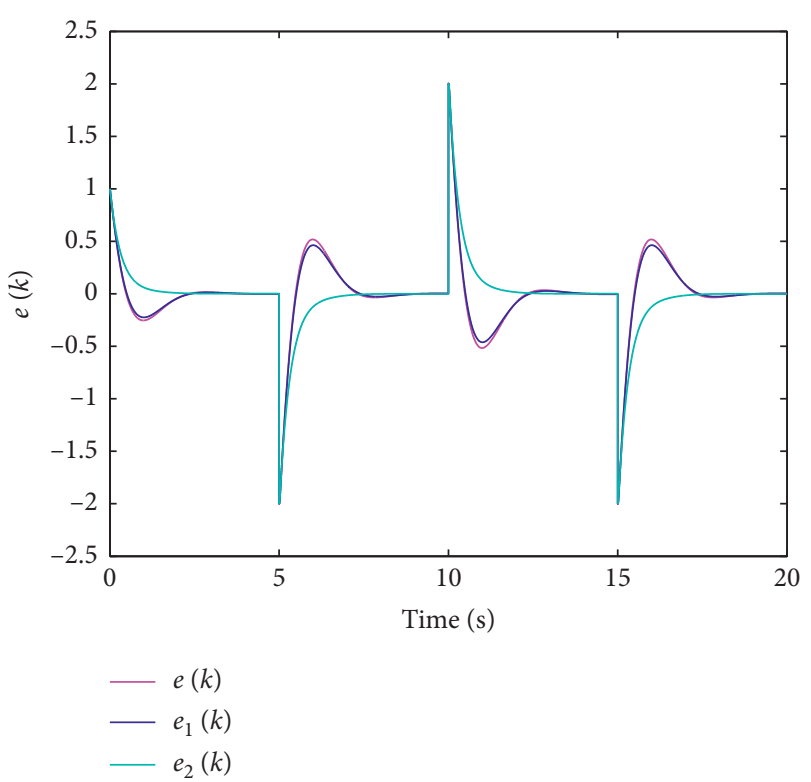

Figure 20: Wave signal tracking error without interference.

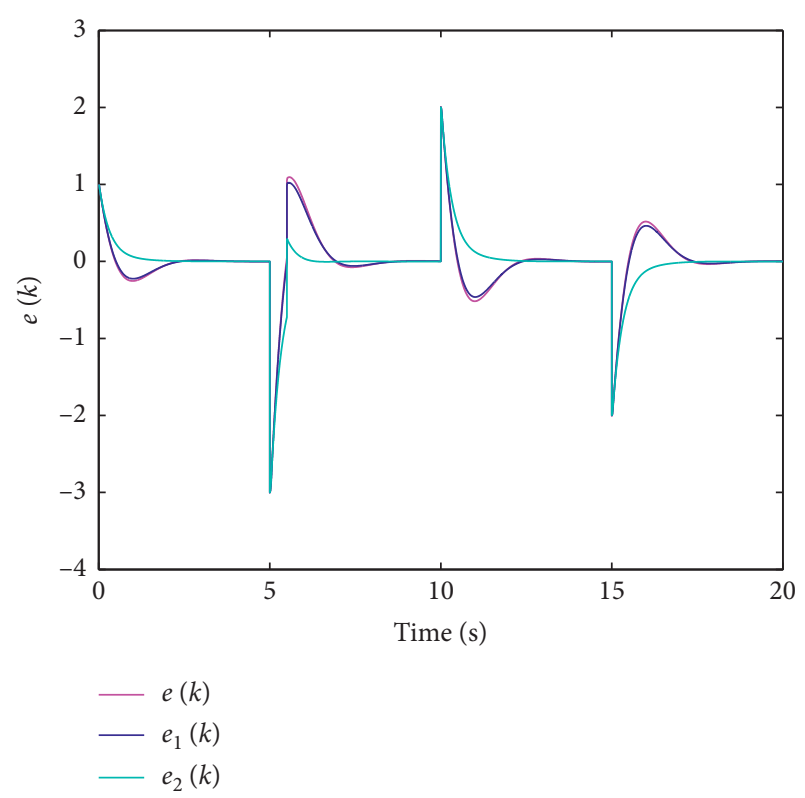

FIgURE 21: Tracking error of wave signal under interference.

other two control algorithms. The IPID-RBF control can reach a stable state in a short time no matter whether there is no interference or interference.

\section{Conclusions}

From the simulation effect, the IPID-RBF control applied to the model has better performance. At the same time, the input signal tracking is well achieved. Under three different input signals, real-time tracking of the input signal is realized through online learning, and the error of the input and output is continuously adjusted, so that the system error eventually approaches zero. Compared with the traditional
PID control, the IPID-RBF control has the best tracking effect with the input signal under three different input signals. It has improved the characteristics of traditional PID with low accuracy. Compared with the PID-RBF supervision control, the IPID-RBF control has smaller curve oscillation amplitude during the adjustment process, and the system reaches a stable state in a short time and has strong anti-interference ability. This algorithm has simple control and good tracking accuracy.

Therefore, the improved control algorithms have good robustness, and the stability of the system is good. Simulation graphics and data show that the IPID-RBF controls the controlled object through online learning to achieve online identification and control. It has high control accuracy, good dynamic characteristics, and anti-interference ability.

\section{Data Availability}

No data were used to support this study.

\section{Conflicts of Interest}

The authors declare that there are no conflicts of interest regarding the publication of this paper.

\section{Acknowledgments}

This work was supported by the National Natural Science Foundation of China under Grants 61971181 and 61602163 and the Natural Science Foundation of Hubei Province under Grant 2016CFC735.

\section{References}

[1] S. M. Attaran, R. Yusof, and H. Selamat, "A novel optimization algorithm based on epsilon constraint-RBF neural network for tuning PID controller in decoupled HVAC system," Applied Thermal Engineering, vol. 99, pp. 613-624, 2016.

[2] Z. Aydin, U. Erol, and Y. Hseyin, "Nonlinear control of an inverted pendulum on a cart by a single control law," Applied Mechanics and Materials, vol. 464, pp. 279-284, 2014.

[3] R. M. Brisilla and V. Sankaranarayanan, "Nonlinear control of mobile inverted pendulum," Robotics and Autonomous Systems, vol. 70, pp. 145-155, 2015.

[4] Z. Chen, X. Yang, and X. Liu, "RBFNN-based nonsingular fast terminal sliding mode control for robotic manipulators including actuator dynamics," Neurocomputing, vol. 362, pp. 72-82, 2019.

[5] Y. Chu, Y. Fang, and J. Fei, "Adaptive neural dynamic global PID sliding mode control for MEMS gyroscope," International Journal of Machine Learning and Cybernetics, vol. 8, no. 5, pp. 1707-1718, 2017.

[6] S. Han, H. Wang, and Y. Tian, "Time-delay estimation based computed torque control with robust adaptive RBF neural network compensator for a rehabilitation exoskeleton," ISA Transactions, vol. 97, pp. 171-181, 2019.

[7] M. Hossein, "Robust predictive control of wheel slip in antilock braking systems based on radial basis function neural network," Applied Soft Computing Journalhttps, vol. 70, pp. 318-329, 2018. 
[8] C. Y. Jia, Y. C. Chen, and Z. G. Ding, "Application in composite machine using RBF neural network based on PID control," Automation Control and Intelligent Systems, vol. 2, no. 6, pp. 100-104, 2014.

[9] F. S. T. Jao, P. C. Tiago, S. Samuel da, and S. R. Jean M de, "Attitude control of inverted pendulums using reaction wheels: comparison between using one and two actuators," Systems and Control Engineering, vol. 234, no. 3, pp. 420-429, 2019.

[10] T. Kunitoshi and N. Sumito, "Posture stability control of a small inverted pendulum robot in trajectory tracking using a control moment gyro," Advanced Robotics, vol. 34, no. 9, 2020.

[11] A. Kharola, P. Dhuliya, and P. Sharma, "Anti-swing and position control of single wheeled inverted pendulum robot (SWIPR)," International Journal of Applied Evolutionary Computation, vol. 9, no. 4, 2018.

[12] A. Wu and Z. Zeng, "Global Mittag-Leffler stabilization of fractional-order memristive neural networks," IEEE Transactions on Neural Networks and Learning Systems, vol. 28, no. 1, pp. 206-217, 2017.

[13] $\mathrm{A}$. Wu and $\mathrm{Z}$. Zeng, "Output convergence of fuzzy neurodynamic system with piecewise constant argument of generalized type and time-varying input," IEEE Transactions on Systems, Man, and Cybernetics: Systems, vol. 46, no. 12, pp. 1689-1702, 2016

[14] A. Wu and Z. Zeng, "Exponential stabilization of memristive neural networks with time delays," IEEE Transactions on Neural Networks and Learning Systems, vol. 23, no. 12, pp. 1919-1929, 2012.

[15] P. Kumar, N. Kumar, and V. Panwar, "RBF neural control design for SISO nonaffine nonlinear systems," Procedia Computer Science, vol. 125, pp. 25-33, 2018.

[16] X. Lian, J. Liu, T. Yuan, and N. Cui, "RBF network based adaptive sliding mode control for solar sails," Aircraft Engineering and Aerospace Technology, vol. 90, no. 8, pp. 1180-1191, 2018.

[17] Z. Li and Y. Bo, "PID adaptive control in the application of the induction motor system based on the RBF neural network inverse," Applied Mechanics and Materials, vol. 2014, pp. 2393-2396, 2014.

[18] C. X. Liu, Z. W. Ping, Y. Z. Huang, J. G. Lu, and H. Wang, "Position control of spherical inverted pendulum via improved discrete-time neural network approach," Nonlinear Dynamics, vol. 2020, 2020.

[19] H. B. Liang, Z. L. Li, and G. L. Li, "Neural network prediction model to achieve intelligent control of unbalanced drillings underpressure value," Springer Nature, vol. 2018, 2018.

[20] P. Liu, H. Yu, and S. Cang, "Adaptive neural network tracking control for underactuated systems with matched and mismatched disturbances," Nonlinear Dynamics, vol. 98, no. 2, pp. 1447-1464, 2019.

[21] P. Morasso, A. Cherif, and J. Zenzeri, "The Single Inverted Pendulum model is not so bad after all," PLoS ONE, vol. 14, no. 3, 2019.

[22] M. J. Mahmoodabadi and H. Khoobroo Haghbayan, "An optimal adaptive hybrid controller for a fourth-order underactuated nonlinear inverted pendulum system," Transactions of the Institute of Measurement and Control, vol. 42, no. 2, pp. 285-294, 2020.

[23] O. C. Oliveira, A. De Medeiros Martins, and A. D. De Araujo, "A dual-mode control with a RBF network," Transactions of the Institute of Measurement and Control, vol. 28, no. 2, pp. 180-188, 2017.
[24] Z. P. Shen, Y. Wang, H. M. Yu, and C. Guo, "Finite-time adaptive tracking control of marine vehicles with complex unknowns and input saturation," Ocean Engineering, vol. 198, 2020.

[25] X. Y. Shi, Y. Y. Cheng, C. Yin, X. G. Huang, and S. M. Zhong, "Design of adaptive back stepping dynamic surface control method with RBF neural network for uncertain nonlinear system," Neurocomputing, vol. 2018, 2018.

[26] F. Wang, Z.-Q. Chao, L.-B. Huang, H.-Y. Li, and C.-Q. Zhang, "Trajectory tracking control of robot manipulator based on RBF neural network and fuzzy sliding mode," Cluster Computing, vol. 22, no. S3, pp. 5799-5809, 2017.

[27] H. Wang, H. R. Karimi, P. X. Liu, and H. Yang, "Adaptive neural control of nonlinear systems with unknown control directions and input dead-zone," IEEE Transactions on Systems, Man, and Cybernetics: Systems, vol. 48, no. 11, pp. 1897-1907, 2018.

[28] H. Wang, P. X. Liu, and B. Niu, "Robust fuzzy adaptive tracking control for nonaffine stochastic nonlinear switching systems," IEEE Transactions on Cybernetics, vol. 48, no. 8, pp. 2462-2471, 2018.

[29] Y. Q. Wang, Q. Lin, X. G. Wang, and F. G. Zhou, “Adaptive PD control based on RBF neural network for a wire-driven parallel robot and prototype experiments," Aircraft Engineering and Aerospace Technology, vol. 2019, 2019.

[30] R. L. Wang, B. C. Lu, and W. B. Ni, "Integral separation PID control of certain electro-hydraulic servo system based on RBF neural network supervision," in Proceedings of the 2nd International Conference on Automation, Mechanical Control and Computational Engineering,(AMCCE 2017), pp. 52-57, Beijing, China, March 2017.

[31] H. Wang, P. Shi, H. Li, and Q. Zhou, "Adaptive neural tracking control for a class of nonlinear systems with dynamic uncertainties," IEEE Transactions on Cybernetics, vol. 47, no. 10, pp. 3075-3087, 2017.

[32] L. L. Wan, Y. X. Su, H. J. Zhang, Y. C. Tang, and B. H. Shi, "Global fast terminal sliding mode control based on radial basis function neural network for course keeping of unmanned surface vehicle," International Journal of Advanced Robotic Systems, vol. 16, no. 2, 2019.

[33] A. Wu and Z. Zeng, "Lagrange stability of memristive neural networks with discrete and distributed delays," IEEE Transactions on Neural Networks and Learning Systems, vol. 25, no. 4, pp. 690-703, 2014.

[34] A. Wu, H. Liu, and Z. Zeng, "Observer design and Ho performance for discrete-time uncertain fuzzy-logic systems," IEEE Transactions on Cybernetics, vol. 1, 2020.

[35] B. Yang, Z.-X. Liu, H.-K. Liu, Y. Li, and S. Lin, “A GPC-based multi-variable PID control algorithm and its application in anti-swing control and accurate positioning control for bridge cranes," International Journal of Control, Automation and Systems, vol. 18, no. 10, pp. 2522-2533, 2020.

[36] A. R. Sergio and F. Gerardo, "PID principles to obtain adaptive variable gains for a Bi-order sliding mode control," International Journal of Control, Automation and Systems, vol. 18, pp. 2456-2467, 2020.

[37] S. Ulusoy, S. M. Nigdeli, and G. Bekda, "Novel metaheuristicbased tuning of PID controllers for seismic structures and verification of robustness," Journal of Building Engineering, vol. 33, 2020.

[38] P. Fišer and J. Fiaer, "A universal map of three-dominant-pole assignment for PID controller tuning," International Journal of Control, vol. 93, no. 9, pp. 2267-2274, 2020.

[39] J.-W. Perng, Y.-C. Kuo, and K.-C. Lu, "Design of the PID controller for hydro-turbines based on optimization 
algorithms," International Journal of Control, Automation and Systems, vol. 18, no. 7, pp. 1758-1770, 2020.

[40] M. H. Zhao, X. B. Xu, H. Yang, and Z. J. Pan, "Design of a predictive RBF compensation fuzzy PID controller for $3 \mathrm{D}$ laser scanning system," Applied Sciences, vol. 10, no. 13, 2020.

[41] J. Zhang, H. C. Sun, Y. M. Qi, and S. P. Deng, "Temperature control strategy of incubator based on RBF neural network PID," World Scientific Research Journal, vol. 6, no. 1, 2020.

[42] M. K. Debnath, R. Agrawal, S. R. Tripathy, and S. Choudhury, "Artificial neural network tuned PID controller for LFC investigation including distributed generation," International Journal of Numerical Modelling: Electronic Networks, Devices and Fields, vol. 33, no. 5, 2020.

[43] Y. W. Wang, W. A. Zhang, H. Dong, and L. Yu, "A LADRC based fuzzy PID approach to contour error control of networked motion control system with time-varying delays," Asian Journal of Control, vol. 22, no. 5, pp. 1973-1985, 2020.

[44] M. Taghdisi and S. Balochian, "Maximum power point tracking of variable-speed wind turbines using self-tuning fuzzy PID," Technology and Economics of Smart Grids and Sustainable Energy, vol. 5, no. 1, pp. 357-366, 2020.

[45] A. Jamali, R. Shahnazi, and A. Maheri, "Load mitigation of a class of 5-MW wind turbine with RBF neural network based fractional-order PID controller Asgharnia," ISA Transactions, vol. 96, pp. 272-286, 2020.

[46] T. K. Mohapatra, A. K. Dey, and B. K. Sahu, "Employment of quasi oppositional SSA-based two-degree-of-freedom fractional order PID controller for AGC of assorted source of generations," IET Generation, Transmission \& Distribution, vol. 14, no. 7, pp. 3365-3376, 2020.

[47] F. Xu, D. Tang, and S. Wang, "Research on parallel nonlinear control system of PD and RBF neural network based on U model," Taylor \& Francis Group, vol. 61, no. 2, pp. 284-294, 2020.

[48] X. Zhao, X. Wang, G. Zong, and H. Li, "Fuzzy-approximation-based adaptive output-feedback control for uncertain nonsmooth nonlinear systems," IEEE Transactions on Fuzzy Systems, vol. 26, no. 6, pp. 3847-3859, 2018. 